\section{Civilizar}

ISSN 1657-8953 e-ISSN: 2619-189X

\title{
División Social por Estratos, Materialismo y la Situación de los Perros en Colombia
}

\author{
Social Division by Strata, Materialism, \\ and the Situation of Dogs in Colombia \\ Gonzalo Luna-Cortés
}

* Artículo de investigación.

$\S$ Doctor en Turismo, Magíster en Marketing e investigación de Mercados. Investigador en la Facultad de Business Administration, en el departamento de Innovation \& Creativity, de la Catholic University of Eichstätt-Ingolstadt, Alemania.

Correo electrónico: Gonzalo.Luna-Cortes@ ku.de.

(iD) $0000-0002-6386-7483$

\section{Cómo citar:}

Luna-Cortés, G. (2019). División Social por Estratos, Materialismo y la Situación de los Perros en Colombia. Civilizar: Ciencias Sociales y Humanas, 19(37) 55-68. doi: 10.22518/usergioa/jour/ ccsh/2019.2/a05

\section{Resumen}

La presente investigación examina una serie de variables que influyen en la preferencia de los dueños de perros de raza en Colombia, así como la forma en que la estratificación social modera esta relación. Se elaboró una investigación de orden cuantitativo, utilizando una encuesta estructurada como herramienta de recogida de información. El trabajo de campo se llevó a cabo en Bogotá. La muestra está formada por dueños de perros de la capital de Colombia. A través de la metodología SEM, test de invarianza y análisis multi-grupo, los resultados muestran que el materialismo influye en la percepción social de los dueños sobre los perros de raza; los perros de dueños más materialistas muestran mayores problemas de comportamiento, lo que influye en la intención de los dueños de abandonarlos. En la mayoría de los casos, estas conductas se presentan en menor intensidad en los ciudadanos de estratos medio y alto que en aquellos que viven en estratos bajos.

\section{Palabras clave}

Materialismo, bienestar de los perros, estratificación social, perros de raza, Bogotá, Colombia.

\section{Abstract}

This paper examines a series of variables that influence the preference of purebred dogs in Colombia, and the role of social stratification regarding this matter. A quantitative methodology was elaborated using a structured questionnaire as data recollection tool. The sample was made up of dog owners in Bogotá, the capital of Colombia, where the fieldwork was conducted. Through the SEM methodology, invariance test and a multiple-group analysis, the results show that materialism influences the social perception about purebred dogs; the dogs of more materialistic owners show greater behavioral problems, which influences owners' intention to abandon them. In most of the cases, these behaviors are less intense in citizens of middle or high strata than in those living in lower strata.

\section{Keywords}

Materialism, dogs' welfare, social stratification system, purebred dogs, Bogotá, Colombia. 


\section{Introducción}

Los perros de compañía son animales altamente valorados en la mayoría de los países del mundo (Farnworth, Blaszak, Hiby y Waran, 2012). Sin embargo, cada sociedad presenta diferentes criterios a la hora de juzgar lo que constituye una vida digna para los perros, y cómo éstos deben ser tratados (Buckland, Abeyesinghe y Wathes, 2014).

En Colombia, los perros callejeros representan un problema importante en términos de sanidad y bienestar. La presente investigación examina la relación entre diferentes variables de comportamiento del consumidor, para observar por qué un número elevado de colombianos prefieren comprar un perro de raza antes que adoptar un perro que ha sido abandonado en un refugio o que se encuentra en la calle. De esta forma, se analizan los siguientes constructos: materialismo, valor social percibido de los perros de raza, problemas comportamentales hallados en dichos perros y la intención de los dueños de abandonar sus mascotas.

Seguido, el estudio se centra en una característica especial de la sociedad colombiana: la estratificación socio-económica implementada por ley en este país, que clasifica las áreas de las ciudades desde las más pobres hasta las más ricas. Se puede observar que el problema de los perros callejeros es aún más notable en los estratos bajos de las ciudades colombianas (Agudelo-Flórez, Castro, Rojo-Ospina y Henao-Villegas, 2012). En este país, existe una necesidad de legislación, inversión y educación con relación al problema comportamental de los perros y el bienestar de estos animales. Esta necesidad se hace aún más patente en las áreas de estrato más bajo de las ciudades (Paternina-Gómez et al., 2013). Por ello, a través de un test de invarianza y un análisis multi-grupo, la presente investigación examina el efecto moderador de la división por estratos en la relación entre el materialismo y el bienestar de los perros en Colombia.

\section{Revisión de la literatura}

\section{Materialismo}

Como se ve reflejado en varias investigaciones en el área de psicología durante el siglo XX, uno de los tópicos de importancia de estudio fue la evolución del consumo con un significado cultural de logro social, felicidad y una noción popular de lo que significa tener una buena vida (Burroughs y Rindfleisch, 2002). Este fenómeno llevó a investigadores de ciencias sociales a analizar el materialismo desde una perspectiva socio-económica (Belk, 1985).

El análisis de este constructo tomó mucha relevancia durante las décadas de los ochenta y los noventa, como un tópico de interés entre investigadores de un gran rango de disciplinas, incluyendo análisis demográficos (Easterlin y Crimmins, 1991), en ciencias políticas (Inglehart, 1990), psicología (Kasser y Ryan, 1993), organización de empresas (Byrne, 1997) y comportamiento del consumidor (Belk, 1985; Richins y Dawson, 1992). Estos estudios forman la base fundamental de la investigación sobre materialismo en la actualidad.

Aunque no todos los autores han examinado el materialismo como un constructo con una connotación meramente negativa (Kilbourne y LaForge, 2010; Kim y Kramer, 2015), las consecuencias perjudiciales del materialismo en sociedad, y a nivel individual, han recibido mayor atención por parte de los académicos durante las últimas décadas (Duh, 2015; Segev, Shoham y Gavish, 2015; Kozinets, Patterson y Ashman, 2016).

Diversos autores han hallado que aquellos individuos que consumen motivados por una actitud o personalidad materialista suelen exhibir patrones de compra impulsiva (Podoshen y Andrzejewski, 2012; Segal y Podoshen, 2012; Harnish y Bridges, 2014). Además, el materialismo puede llevar a niveles más reducidos de satisfacción en la vida del individuo (Richins y Dawson, 1992; Sirgy et al., 1995; Richins y Chaplin, 2015), disminuyendo la felicidad (Ergen, Baykan y Turan, 2015; Segev, Shoham y Gavish, 2015) y generando un mayor riesgo de trastornos psicológicos (Deckop, Jurkiewicz y Giacalone, 2010; Harnish y Bridges, 2014; Dogan, 2015).

Aunque existe una carencia de análisis empíricos para poder afirmar que el materialismo influye en actitudes negativas hacia perros que no son de raza, existe evidencia científica que nos facilita plantear esta hipótesis. Se observa en la literatura que los consumidores materialistas presentan actitudes más cínicas que la media poblacional, además de ser un comportamiento que se asocia con el racismo (Roets, Van Hiel y Cornelis, 2006) y con otro tipo de prejuicios (Van Hiel, Cornelis y Roets, 2010; Segal y Podoshen, 2012; Veer y Shankar, 2011); los hábitos de consumo de los materialistas también se relacionan en la literatura con formas poco éticas (Burroughs y Rindfleisch, 2002); además, el materialismo influye de manera negativa en la preocupación de los ciuda- 
danos por el bienestar público general (Burroughs y Rindfleisch, 2002; Segal y Podoshen, 2012).

\section{La adquisición de perros en el mercado y el bienestar animal}

Los perros de compañía suelen exhibir una gran variedad de comportamientos que los dueños pueden considerar molestos. Estas conductas van desde problemas menores, como observar al perro tirando demasiado de la cadena, a problemas mucho más serios, como la agresividad (Luna-Cortés, 2018a; Wells y Hepper, 2000).

Los motivos de comportamientos molestos pueden ser la raza del perro, el género, la edad, si ha sido castrado, su dieta, etc. (Wells y Hepper, 2000). De acuerdo con Buckland et al. (2014), las razones principales de los problemas comportamentales en los perros son una crianza y educación inadecuadas, la carencia de conocimiento de los dueños y una falta de socialización. Así como Ellingsen, Zanella, Bjerkas e Indrebo (2010) afirman que el nivel de empatía y la actitud hacia la mascota son los factores más influyentes en la relación entre el humano y el animal.

En la actualidad, se estima que más del 9o\% de los perros muestran comportamientos que los dueños consideran inaceptables (Vacalopoulos y Anderson, 1993; Wells y Hepper, 2000). Para muchos, la única solución que encuentran es abandonar al perro en un refugio para animales (Patronek, Glickman y Moyer, 1995; Salman, New, Scarlett, Kass, Ruch-Gallie y Hetts, 1998). Más del 30\% de los perros que llegan a estos refugios son abandonados debido a que los dueños perciben problemas comportamentales en el animal (Wells y Hepper, 2000).

Algunos factores relacionados con la forma de adquirir los perros en el mercado se mencionan en la literatura como variables que influyen en el comportamiento de estos animales. Entre éstos, se indican las condiciones en las que se encuentran los perros en las tiendas, bajo el cuidado de personal a veces no cualificado y retenidos en espacios demasiado pequeños, (King, Marston y Bennett, 2012; Sonntag y Overall, 2014). De esta forma, algunos autores sugieren que los perros adquiridos en tiendas de animales presentan más problemas comportamentales que aquellos que son obtenidos a través de amigos, familiares o criadores especializados (Wells y Hepper, 2000).
A ello hay que añadir que estos animales pueden sufrir si se crían con el único objetivo de conservar las características meramente físicas de una determinada raza, debido a que los estándares requeridos pueden llevar a conservar también enfermedades o problemas congénitos (McGreevy y Nicholas, 1999; Rooney y Sargan, 2010). De hecho, está comprobado que los perros criollos presentan menos costos médicos que los perros con pedigrí (Rooney y Sargan, 2010).

Muchos prejuicios sobre el animal influyen a la hora de elegir un perro (Wright, Smith, Daniel y Adkins, 2007). En este sentido, las razas que se pueden relacionar con estatus social tienen una mejor aceptación entre algunas personas (King, Marston y Bennett, 2012; Sonntag y Overall, 2014).

Reese (2005) señaló que "en algunos países en vías de desarrollo, los perros se compran más por el estatus que representan que por sus atributos como animal de compañía” (p. 55). En la investigación de Wright et al. (2007), los autores indican que los dueños potenciales tienden a atribuir valor al animal con base en información previa sobre determinados perros, teniendo en cuenta los estereotipos que marcan a las diferentes razas. Tanto los estereotipos como el estatus social son variables que juegan un rol importante en las investigaciones de consumo simbólico en general $\mathrm{y}$, particularmente, en los estudios sobre comportamientos materialistas (Kim y Jang, 2014).

\section{La estratificación social en Colombia}

En los años ochenta se estableció un sistema de estratificación social en Colombia, el cual clasifica de 1 a 6 las urbes en zonas con características económicas similares. Los estratos 1,2 y 3 se consideran de bajo nivel económico; el estrato 4 es considerado de nivel económico medio; el estrato 5 se considera estrato medio-alto; y el estrato 6 es el más alto de Bogotá. En 1994 este sistema adoptó carácter legal en las ciudades de todo el territorio Colombiano (Medina, Morales, Bernal y Torero, 2007).

Aunque el sistema de estratificación fue diseñado para ayudar a los más pobres, no deja de estigmatizar a las personas de estrato más bajo, quienes son conscientes de que solo las personas de estrato medio o alto tendrán la oportunidad de conseguir establecerse en los estratos más altos de Bogotá. Una clara disparidad en las inversiones públicas, en la que se observa que los mejores transportes, 
parques, zonas de recreo o las mejores escuelas se encuentran en los estratos 4, 5 y 6, donde, además, el coste de vida crece cada año (Bogliacino, Jiménez-Lozano y Reyes-Galvis, 2015).

Algunos autores colombianos consideran que las personas que viven en estratos bajos de Bogotá perciben la exclusión y discriminación social como algo normal (Gallego, López y Sepúlveda, 2014), ya que, aunque las diferencias de estatus económicos son evidentes en todas las sociedades del mundo, hay pocos países que categorizan a sus ciudadanos de manera explícita a través de una política pública (Uribe-Mallarino, 2008).

En general, las personas de bajo estatus social tienen menos recursos (Drentea, 2000; Oakes y Rossi, 2003) a la par que menos posibilidades de formarse para la vida laboral (Snibbe y Markus, 2005). Además, suelen tener que enfrentarse a situaciones complicadas durante sus vidas (Gallo et al., 2005), como por ejemplo, mayores índices de violencia dentro y fuera del hogar (Staggs, Long, Mason, Krishnan y Riger, 2007). Por ello, algunos autores indican que las personas de estrato social bajo se ven obligadas a centrarse en su propio bienestar, por lo que priorizan sus necesidades sobre el bienestar general de su ciudad (Piff, Kraus, Côté, Hayden y Keltner, 2010).

En la relación de las clases sociales con el comportamiento de los consumidores, se observa que los niveles económicos influyen en la percepción y los valores de los individuos al comprar (Amaral y Loken, 2016; Carey y Markus, 2016). La mayoría de las investigaciones se centran en la influencia de este tipo de segmentación en el consumo de determinados bienes (Amaral y Loken, 2016) y experiencias (Shavitt, Jiang y Cho, 2016). Sin embargo, existe una escasez de literatura en relación a la influencia de la segmentación por clases sociales y el comportamiento de quienes adquieren mascotas.

En Colombia, el problema de los perros abandonados es más notable en los estratos bajos de las ciudades (Ruíz, Giraldo y López, 2010; Agudelo-Flórez et al., 2012; Paternina-Gómez, Díaz-Olmos, Paternina y Bejarano, 2013). Por ejemplo, las investigaciones de Ruíz et al. (2010) y de Agudelo-Flórez et al. (2012) muestran que los perros callejeros que se encuentran en las áreas pobres de Medellín generan serios problemas relacionados con la reproducción de enfermedades. Las mismas conclusiones se observan en los estudios de Paternina-Gómez et al. (2013) en la ciudad de Cartagena de Indias; de Pardo, Pérez, Góngora, Gómez y Moreno (2009) en Villavicencio y de Fernández et al. (2006), en la ciudad de Neiva.

\section{Planteamiento de Hipótesis}

\section{El consumismo y el valor social percibido en los perros de raza}

La mayoría de los estudios sobre materialismo se centran en el consumo de productos y servicios. No obstante, algunos consumidores adquieren perros prestando especial atención a cómo sus atributos pueden mostrar estatus social (King, Marston y Bennett, 2012; Sonntag y Overall, 2014). También se ha mencionado en la literatura que los dueños se centran en los estereotipos que reflejan las razas a la hora de elegir su perro (Wright et al., 2007).

De este modo, algunos dueños (o dueños potenciales) evalúan los atributos del perro en función del estatus que representa (tal y como evalúan las marcas en el mercado). Buscar estatus social a través de las posesiones es uno de los elementos más importantes en relación al constructo materialismo (Belk, 1985). Además, el materialismo influye en el valor social percibido de las posesiones materiales (Richins y Dawson, 1992). Con base en estas afirmaciones, se plantea la siguiente hipótesis:

H1: El consumismo influye en la percepción social de los perros de raza.

\section{Consumismo y problemas comportamentales de los perros}

Cuando los consumidores adquieren perros basándose en estereotipos u otras razones de índole social, dichos perros pueden presentar mayores problemas de salud y comportamiento (Wells y Hepper, 2000). Se puede plantear que estos problemas surgen con mayor probabilidad si las mascotas son obtenidas como objetos o productos que cubren las necesidades sociales del consumidor. De esta forma, los individuos pueden anteponer sus necesidades sociales a las necesidades del animal, lo que puede conllevar problemas de comportamiento observados en el perro. A raíz de estas afirmaciones, se plantean las siguientes hipótesis:

H2: El consumismo influye en los problemas comportamentales de los perros de raza. 
H3: El valor social percibido de los perros de raza influye en los problemas comportamentales de estos animales.

\section{Materialismo, valor social percibido e intención de abandonar a la mascota}

Para muchos dueños, la solución que encuentran al enfrentar problemas comportamentales de sus mascotas es dejarlas en un refugio (Patronek et al., 1995; Salman et al., 1998; Wells y Hepper, 2000). Durante la revisión de la literatura se mencionó que los dueños que se enfrentan a problemas comportamentales de sus perros son más propensos a abandonarlos (Wells y Hepper, 2000). De este modo:

H4: Los problemas comportamentales del perro influyen en la intención de abandono por parte del dueño.

Por otro lado, los consumidores más materialistas suelen sentir la necesidad impulsiva de consumo de determinados bienes, lo cual los lleva a un rápido aburrimiento o insatisfacción con el producto (Belk, 1985). Así, las personas materialistas tienden a deshacerse de sus posesiones de forma más rápida (Podoshen y Andrzejewski, 2012; Rose, Bakir y Gentina, 2016; Segal y Podoshen, 2012; Harnish y Bridges, 2014). A todo ello, se puede añadir que las personas más materialistas podrán sentir menos malestar psicológico por el hecho de deshacerse de su mascota, ya que se estableció que el materialismo influye negativamente en la conciencia cívica de los consumidores en relación al bienestar de la sociedad (Burroughs y Rindfleisch, 2002; Segal y Podoshen, 2012). Basado en estos estudios previos, se plantean las siguientes hipótesis:

H5: El valor social percibido de los perros de raza influye en la intención de abandono de este tipo de mascotas.

H6: El materialismo influye en la intención de abandono de los perros de raza.

\section{El efecto moderador de la estratificación social en el bienestar de los perros}

Como se mencionó anteriormente, la estratificación social divide a las ciudades colombianas en zonas ricas y pobres. Estudios previos han mostrado que esta categorización social se correlaciona con los niveles de educación, de recursos y de percepción de peligros por parte de los ciudadanos (Medina et al., 2007; Bogliacino, Jiménez-Lozano y Reyes-Galvis, 2015; Gallego, López y Sepúlveda, 2014).
Investigaciones recientes documentan que la división social influye en patrones cognitivos y conductuales (Amaral y Loken, 2016; Carey y Markus, 2016; Kraus et al., 2009). Dichos patrones pueden tener una influencia en la percepción y comportamiento de las personas en relación a la adquisición y protección de los perros. De hecho, a raíz de sus propias circunstancias, los ciudadanos con menos recursos suelen priorizar sus propias necesidades, por encima de otras cuestiones de interés social (Piff et al., 2010). Ello podría verse reflejado en la priorización de las necesidades propias por encima de las necesidades de los perros abandonados en su ciudad. Basados en estas premisas, se plantea la siguiente hipótesis:

H7: La estratificación social modera las relaciones estructurales entre el materialismo, el valor social percibido, los problemas de comportamiento de los perros y la intención de abandonarlos.

La hipótesis 7 siguiere que existen variaciones en las relaciones planteadas, a tenor de la variable moderadora "estratificación social". Así, dicha hipótesis puede ser analizada a partir de una serie de sub-hipótesis:

H7a: La relación entre el materialismo y el valor social percibido de los perros de raza es moderada por la estratificación social en Colombia.

H7b: La relación entre el materialismo y los problemas comportamentales de los perros de raza es moderada por la estratificación social en Colombia.

H7c: La relación entre el valor social percibido de los perros de raza y los problemas comportamentales es moderada por la estratificación social en Colombia.

H7d: La relación entre los problemas comportamentales y la intención de abandonar a la mascota es moderada por la estratificación social en Colombia.

H7e: La relación entre el valor social percibido de los perros de raza y la intención de abandonar la mascota es moderada por la estratificación social en Colombia.

H7f: La relación entre el materialismo y la intención de abandonar a la mascota es moderada por la estratificación social en Colombia. 
La Figura 1 muestra de forma gráfica las hipótesis planteadas en esta investigación:

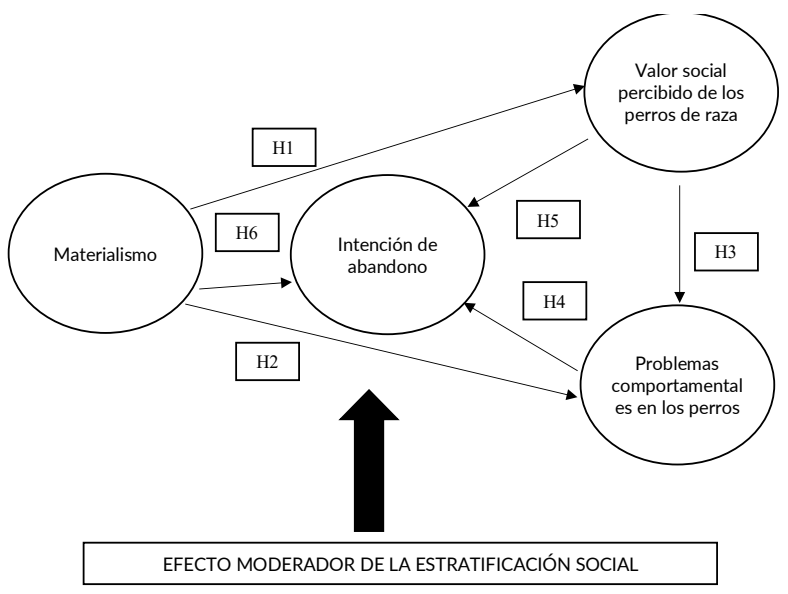

Figura 1. Modelo teórico. Fuente: Elaboración propia.

\section{Metodología}

Para obtener los datos y examinar las hipótesis planteadas, se hizo un estudio de corte cuantitativo, utilizando una encuesta estructurada como herramienta para recolectar información. La muestra se obtuvo a partir de dueños de perros de raza de la ciudad de Barranquilla (Colombia). Los perros debían haber sido comprados para que los dueños pudiesen responder la encuesta. Es decir, ninguno de los encuestados había adoptado al animal en un refugio o lo había acogido de la calle. El método de muestreo fue por conveniencia. Las encuestas se aplicaron en parques donde los ciudadanos llevan sus perros a pasear. Como resultado, se obtuvieron 355 encuestas válidas durante el primer cuatrimestre del año 2015.

El cuestionario estaba formado por las escalas de medición de los constructos que se analizan en esta investigación. La escala Durvasula y Lysonski (2010) se utilizó para medir el constructo materialismo; la escala de Sweeney y Soutar (2001) se adaptó para medir el valor social percibido de los perros de raza; una escala de seis ítems se construyó para medir la percepción de los dueños sobre los problemas comportamentales de sus perros. Esta escala se basó en los factores del cuestionario de Hsu y Serpell (2003). Finalmente, la escala de intención de abandonar al perro es una escala creada a partir de una serie de estudios obtenidos en la literatura sobre intención comportamental (véanse Taylor y Todd, 1995; Karahanna y Straub, 1999; Kuo y Yen, 2009). Esta escala está formada por tres ítems que miden la posibilidad de que los dueños abandonen a su perro en un futuro cercano. La Tabla 1 muestra los ítems del cuestionario.

Las últimas preguntas del cuestionario estaban dirigidas a recopilar las características socio-demográficas de la muestra. Los encuestados dieron información sobre su género, edad, nivel de estudios, estado civil y el estrato en el que residen. De acuerdo con estas respuestas, el $59 \%$ de los

Tabla 1.

Escalas e ítems del cuestionario

\begin{tabular}{|c|c|c|}
\hline Variable & Escala & Ítems \\
\hline Materialismo & $\begin{array}{l}\text { Durvasula } \\
\text { y Lysonsky } \\
(2010)\end{array}$ & $\begin{array}{l}\text { 1. Para mí es importante poseer cosas que sean realmente } \\
\text { bonitas. } \\
\text { 2. Me gustaría poder ser rico para comprar todo lo que quiera. } \\
\text { 3. Sería más feliz si pudiera comprar más cosas. } \\
\text { 4. A veces me molesta no poder comprar todas las cosas que } \\
\text { me gustaría tener. }\end{array}$ \\
\hline $\begin{array}{l}\text { Valor social } \\
\text { percibido }\end{array}$ & $\begin{array}{l}\text { Sweeney } \\
\text { y Soutar } \\
\text { (2001) }\end{array}$ & $\begin{array}{l}\text { 1. Mi perro hace que me sienta aceptado. } \\
\text { 2. Mi perro mejora la forma en cómo me ven. } \\
\text { 3. Mi perro genera una gran impresión en otras personas. } \\
\text { 4. Mi perro me otorga aprobación social. }\end{array}$ \\
\hline $\begin{array}{l}\text { Problemas } \\
\text { comportamentales } \\
\text { en los perros }\end{array}$ & $\begin{array}{l}\text { Hsu and } \\
\text { Serpell } \\
(2003)\end{array}$ & $\begin{array}{l}\text { 1. Mi perro es agresivo con extraños. } \\
\text { 2. Mi perro se asusta fácilmente, lo cual es un problema. } \\
\text { 3. Mi perro no es sociable. } \\
\text { 4. Mi perro se pone ansioso fácilmente y en muchas ocasiones. }\end{array}$ \\
\hline $\begin{array}{l}\text { Intención de } \\
\text { abandonar al perro }\end{array}$ & $\begin{array}{l}\text { Elaboración } \\
\text { propia }\end{array}$ & $\begin{array}{l}\text { 1. Existe la posibilidad de que le de mi perro a alguien. } \\
\text { 2. Tal vez, en el futuro, busque una solución con mi perro, } \\
\text { porque no puedo tenerlo más. } \\
\text { 3. Es posible que me deshaga de mi perro. }\end{array}$ \\
\hline
\end{tabular}

Fuente: Elaboración propia. 
encuestados fueron mujeres y el $41 \%$ hombres. La edad de los encuestados varió bastante, pero el $85 \%$ de la muestra tenía entre 35 y 50 años. El 54\% eran casados, el $44 \%$ no estaban casados, pero tenían una pareja sentimental con la cual compartía el perro. Solo tres encuestados de los 355 no tenían pareja.

Para fines de la investigación, los encuestados fueron divididos en dos grupos. El primero, de personas que residen en estratos bajos de la ciudad (estratos 1, 2 y 3); y el segundo, de personas que residen en estratos altos de la ciudad (estratos 4, 5 y 6). De acuerdo con esta división, el $49 \%$ de los encuestados vive en áreas oficialmente categorizadas como estratos bajos, mientras que el $51 \%$ vive en estratos medios y altos de la ciudad.

$\mathrm{El}$ 53\% de los encuestados había terminado sus estudios universitarios. De acuerdo con estos datos, se observa una asociación entre el nivel educativo y el estrato en el que vive el encuestado. Así, solo el 32\% de los individuos encuestados que viven en estratos bajos tiene un título universitario, por lo que la mayoría de este $53 \%$ que tiene un título universitario viven en estratos altos de la ciudad.

En relación con el método de análisis de datos, antes de testear las hipótesis, se verificó la fiabilidad y validez de las escalas utilizadas en el estudio, a través de un análisis factorial confirmatorio y el uso del software SPSS-AMOS de IBM, versión 20. Posteriormente, se empleó el método de ecuaciones estructurales con el mismo programa, con el fin de examinar los coeficientes de las relaciones causales entre los constructos que forman el modelo teórico de la investigación. Finalmente, se aplicó un test de invarianza y un análisis multi-grupo para examinar el efecto moderador de la estratificación social en las relaciones planteadas. De la misma forma, estos análisis se llevaron a cabo a través de SPSS-AMOS de IBM, versión 20.

\section{Resultados}

\section{Validación del instrumento de medida}

En primer lugar, se debe mencionar que el modelo en su conjunto no mostraba una bondad de ajuste aceptable, pues los índices de bondad de ajuste mostraban valores más bajos que los recomendados en la literatura (Hatcher, 1994). Para solucionar este problema, los ítems de las escalas fueron revisados. Se pudo observar que el segundo ítem de la escala materialismo (MAT2), el primero de la escala problemas comportamentales (PC1) y el último ítem de la escala valor social percibido (VSOCIAL4) arrojaban cargas factoriales mucho más bajas que el resto de los ítems en sus correspondientes constructos. Además, se revisaron los estadísticos descriptivos de los ítems de las escalas, y se observó que estos ítems mostraban medias significativamente diferentes al resto de los ítems de sus escalas.

Se procedió a aplicar el test de Lagrange y, aunque este no sugería cambio de ítems entre las

Tabla 2.

Fiabilidad Compuesta y Validez

\begin{tabular}{|c|c|c|c|c|c|c|}
\hline Factor & Indicador & Carga & T-Student & a Cronbach & $\begin{array}{l}\text { Fiabilidad } \\
\text { compuesta }\end{array}$ & $\begin{array}{l}\text { Varianza } \\
\text { extraída } \\
\text { promedio }\end{array}$ \\
\hline Materialismo & $\begin{array}{l}\text { MAT1 } \\
\text { MAT3 } \\
\text { MAT4 }\end{array}$ & $\begin{array}{l}0,608^{* *} \\
0,710^{* *} \\
0,767^{* *}\end{array}$ & $\begin{array}{l}12,005 \\
12,578 \\
14,219\end{array}$ & 0,728 & 0,738 & 0,581 \\
\hline Valor social & $\begin{array}{l}\text { SOCVAL1 } \\
\text { SOCVAL2 } \\
\text { SOCVAL3 }\end{array}$ & $\begin{array}{l}0,812^{* *} \\
0,777^{* *} \\
0,698^{* *}\end{array}$ & $\begin{array}{l}14,889 \\
15,614 \\
14,364\end{array}$ & 0,742 & 0,807 & 0,710 \\
\hline $\begin{array}{l}\text { Problemas } \\
\text { comportamentales }\end{array}$ & $\begin{array}{l}\text { PC2 } \\
\text { PC3 } \\
\text { PC4 }\end{array}$ & $\begin{array}{l}0,707^{* *} \\
0,838^{* *} \\
0,775^{* *}\end{array}$ & $\begin{array}{l}11,103 \\
14,111 \\
12,720\end{array}$ & 0,814 & 0,818 & 0,730 \\
\hline $\begin{array}{l}\text { Intención de } \\
\text { abandono }\end{array}$ & $\begin{array}{l}\text { ABAND1 } \\
\text { ABAND2 } \\
\text { ABAND3 }\end{array}$ & $\begin{array}{l}0,675^{* *} \\
0,802^{* *} \\
0,701^{* *}\end{array}$ & $\begin{array}{l}11,707 \\
12,195 \\
10,924\end{array}$ & 0,765 & 0,770 & 0,642 \\
\hline \multicolumn{7}{|c|}{$N=355 ;{ }^{* *} p<0,01 ; S-B \times 2=182,3, g I=48 ; N F I=0,894 ; G F I=0,924 ; C F I=0,918 ; I F I=0,919$} \\
\hline
\end{tabular}


escalas, sí sugería, precisamente, la eliminación de estos tres ítems para mejorar la bondad de ajuste del modelo. Por lo tanto, fueron descartados. Después de estos cambios, se obtuvo una bondad de ajuste aceptable para continuar con el análisis, tal y como se puede observar en la última fila de la Tabla 2.

La Tabla 2 muestra la validez convergente que puede ser aceptada, ya que todos los ítems mostraban cargas factoriales superiores a o,6o (Bagozzi y Yi, 1988). La fiabilidad fue medida a través del Alphas de Cronbach. El valor mínimo para aceptar fiabilidad a tenor de este estadístico es 0,70, lo cual garantiza consistencia entre los ítems de las escalas (Nunnally y Bernstein, 1994). Puede observarse que todos los Alphas de Cronbach presentan valores superiores a 0,70. La fiabilidad compuesta también fue calculada, superando para todas las escalas el valor recomendado de o,70 (Fornell y Larcker, 1981). Además, el análisis de la varianza extraída prome- dio presentó valores superiores a 0,50 para todas las escalas, lo cual es lo que se recomienda en la literatura para aceptar fiabilidad a partir de este índice (Fornell y Larcker, 1981).

La validez discriminante fue evaluada siguiendo dos criterios (véase la Tabla 3). Primero, se verificó que ninguno de los intervalos de confianza creados a partir de las correlaciones estimadas para cada par de constructos superase el valor uno. En segunda instancia, se examinó que las correlaciones al cuadrado para cada par de constructos fuera en todo caso menor a sus varianzas extraídas promedio, previamente calculadas (Fornell y Larcker, 1981).

\section{Análisis de Ecuaciones estructurales}

La Tabla 4 muestra la verificación de las hipótesis para el conjunto de la muestra. Se puede observar que cinco de las seis hipótesis planteadas pueden ser aceptadas.

Tabla 3.

Validez Discriminante

\begin{tabular}{|l|r|r|r|r|}
\hline & Materialismo & Valor social & $\begin{array}{c}\text { Problemas } \\
\text { Comportamentales }\end{array}$ & $\begin{array}{c}\text { Intención de } \\
\text { abandono }\end{array}$ \\
\hline Materialismo & $\mathbf{0 , 5 8 1}$ & $(0,28-0,59)$ & $(0,26-0,49)$ & $(0,30-0,59)$ \\
\hline Valor social & 0,190 & 0,710 & $(0,41-0,75)$ & $(0,21-0,58)$ \\
\hline $\begin{array}{l}\text { Problemas } \\
\text { comportamentales }\end{array}$ & 0,142 & 0,341 & 0,730 & $(0,36-0,67)$ \\
\hline Intención de abandono & 0,197 & 0,156 & 0,268 & $\mathbf{0 , 6 4 2}$ \\
\hline
\end{tabular}

Nota: la diagonal presenta la varianza extraída promedio. Por debajo de la diagonal se presenta la correlación al cuadrado para cada par de constructos. Por encima de la diagonal se presenta el intervalo de confianza al 95\% para las correlaciones entre factores. Fuente: elaboración propia.

Tabla 4.

Verificación de las hipótesis

\begin{tabular}{|c|c|c|c|}
\hline Relación & $\boldsymbol{\beta}$ & $(\mathrm{t})$ & Hipótesis \\
\hline $\mathrm{H} 1:$ Consumismo $\rightarrow$ Valor social percibido & $0,459^{* *}$ & 6,155 & Aceptada \\
\hline H2: Consumismo $\rightarrow$ Problemas comportamentales en el perro & $0,463^{* *}$ & 5,796 & Aceptada \\
\hline H3: Valor social percibido $\rightarrow$ Problemas comportamentales en el perro & $0,322^{* *}$ & 4,702 & Aceptada \\
\hline H4: Problemas comportamentales en el perro $\rightarrow$ Intención de abandono & $0,407^{* *}$ & 4,373 & Aceptada \\
\hline H5: Valor social percibido $\rightarrow$ Intención de abandono & $-0,090^{* *}$ & $-1,216$ & No aceptada \\
\hline H6: Materialismo $\rightarrow$ Intención de abandono & $0,368^{* *}$ & 3,969 & Aceptada \\
\hline
\end{tabular}

Notas: los dueños más materialistas perciben un valor social mayor de los perros de raza que los individuos menos materialistas (H1); tanto el materialismo como el valor social percibido de los perros de raza influye en los problemas comportamentales que muestran estos animales ( $\mathrm{H} 2$ y H3); los dueños que perciben mayores problemas comportamentales en sus perros muestran una mayor intención de abandonarlos (H4); y el materialismo influye directamente en la intención de los dueños de abandonar a sus mascotas (H6). Sin embargo, no se pudo demostrar que el valor social percibido de los perros de raza influye en la intención de abandonar a las mascotas (H5). Fuente: elaboración propia. 


\section{Test de invarianza y análisis multi-grupo}

El test de invarianza se aplicó para aquellas hipótesis del modelo que fueron aceptadas. Así, los encuestados fueron categorizados en dos grupos, según el estrato en el que residen (bajo y medio/ alto). Como sugieren Dabholkar y Bagozzi (2002), si se observa un cambio significativo en la Chi-cuadrado entre el modelo contraído y el modelo sin contraer, se puede afirmar que existe un efecto de la variable moderadora en el modelo.

Con base en estas diferencias, se pasó a examinar en cuáles relaciones se observa un efecto moderador de la variable división por estratos. En la Tabla 6 se presentan diferencias significativas en las relaciones del modelo de acuerdo con esta división. Así, la relación entre materialismo y valor social percibido solo puede ser aceptada para los dueños de perros que residen en los estratos 1,2 y 3 de la ciudad. Para aquellos dueños de perros que viven en los estratos 4, 5 y 6 esta relación no puede ser acep- tada de acuerdo con los resultados del análisis. Se observa el mismo efecto en la relación entre el valor social percibido de los perros de raza y los problemas comportamentales de las mascotas. Esta relación solo puede ser aceptada para aquellos individuos que viven en los estratos más bajos de la ciudad. Finalmente, la relación entre dueños que perciben problemas comportamentales en sus mascotas y la intención de abandonarlos puede ser aceptada para ambos grupos, no obstante, se observa una relación mucho más fuerte entre los ciudadanos que viven en los estratos 1,2 y 3 que en los ciudadanos que residen en los estratos 4,5 y 6 .

\section{Conclusiones}

La presente investigación establece que el materialismo tiene un efecto sobre el valor social percibido de los perros de raza. Así, los colombianos dueños de perros más materialistas dan mayor importancia a los perros de raza, con relación al valor que este tipo de mascotas exhiben en socie-

Tabla 5.

Efecto moderador

\begin{tabular}{|l|r|r|r|r|r|r|r|r|}
\hline & $\begin{array}{c}\text { Chi- } \\
\text { cuadrado }\end{array}$ & gl & GFI & CFI & $\begin{array}{c}\text { Diferencias } \\
\text { en la Chi- } \\
\text { cuadrado }\end{array}$ & $\begin{array}{c}\text { Diferencias } \\
\text { en los gl }\end{array}$ & p-valor \\
\hline $\begin{array}{l}\text { Modelo } \\
\text { básico }\end{array}$ & 182,3 & 48 & 0,924 & 0,918 & & \\
\hline $\begin{array}{l}\text { Variable } \\
\text { moderadora }\end{array}$ & Sin contraer & 268,9 & 97 & 0,886 & 0,848 & & \\
\hline & Contraído & 317,11 & 111 & 0,874 & 0,837 & 48,21 & 14 & 0,000 \\
\hline
\end{tabular}

Notas: La variable moderadora, división por estratos, genera un cambio significativo en la Chi-cuadrado y los grados de libertad. Fuente: elaboración propia.

Tabla 6.

Comparación entre las hipótesis para estratos altos y bajos

\begin{tabular}{|c|c|c|c|c|c|c|c|}
\hline \multirow[t]{2}{*}{ Hipótesis } & \multicolumn{2}{|c|}{ Estratos Medios y altos } & \multirow[b]{2}{*}{$\begin{array}{c}\text { Hipótesis para } \\
\text { este grupo }\end{array}$} & \multicolumn{2}{|c|}{ Estratos bajos } & \multirow[b]{2}{*}{$\begin{array}{l}\text { Hipótesis para } \\
\text { este grupo }\end{array}$} & \multirow[t]{2}{*}{ Comparación } \\
\hline & $\boldsymbol{\beta}$ & $(t)$ & & $\boldsymbol{\beta}$ & $(t)$ & & \\
\hline $\begin{array}{l}\text { Materialismo } \rightarrow \\
\text { Valor social }\end{array}$ & $0,127^{* *}$ & 0,278 & No aceptada & $0,343^{* *}$ & 0,002 & Aceptada & Bajo > Alto \\
\hline $\begin{array}{l}\text { Materialismo } \\
\rightarrow \text { Problemas } \\
\text { comportam. }\end{array}$ & $0,557^{* *}$ & 0,000 & Aceptada & $0,407^{* *}$ & 0,000 & Aceptada & Bajo = Alto \\
\hline $\begin{array}{l}\text { Valor social } \\
\rightarrow \text { Problemas } \\
\text { comportam. }\end{array}$ & $0,117^{* *}$ & 0,256 & No aceptada & $0,389^{* *}$ & 0,000 & Aceptada & Bajo > Alto \\
\hline $\begin{array}{l}\text { Problemas } \\
\text { comportam. } \rightarrow \\
\text { Abandono }\end{array}$ & $0,197^{* *}$ & 0,015 & Aceptada & $0,463^{* *}$ & 0,000 & Aceptada & Bajo > Alto \\
\hline $\begin{array}{l}\text { Materialismo } \\
\rightarrow \text { Abandono }\end{array}$ & $0,360^{* *}$ & 0,109 & Aceptada & $0,259^{* *}$ & 0,022 & Aceptada & Bajo = Alto \\
\hline
\end{tabular}

Fuente: elaboración propia. 
dad. Además, se observa que ellos son quienes más manifiestan problemas de comportamiento en sus mascotas presentan mayores problemas de comportamiento (Luna-Cortés, 2018b). Al mismo tiempo, aquellos dueños que otorgan un mayor valor social a sus perros de raza también perciben mayores problemas de comportamiento en sus mascotas. Estos problemas influyen en la intención de los dueños de deshacerse de sus perros, a lo que hay que añadir que los más materialistas muestran una mayor intención de abandonar a sus animales que los menos materialistas.

Así, puede afirmarse que algunos colombianos piensan antes en sus propias necesidades que las de sus mascotas cuando adquieren un perro de raza. Esta forma de adquirir un animal influye en los problemas comportamentales de estos animales. Finalmente, cuando los dueños perciben comportamientos indeseados, algunos piensan en abandonarlos como solución del problema.

A raíz de estos resultados, se puede sugerir que las instituciones públicas de Colombia deben hacer un esfuerzo para incrementar la conciencia pública, sobre las consecuencias que tiene comprar una mascota basándose en el estatus social que el animal representa. Parece importante que los ciudadanos entiendan que los perros son seres vivos con necesidades que deben ser cubiertas; de no ser así, estos animales suelen presentar problemas de comportamiento que dificultan la vida del dueño.

Por otro lado, en Colombia hay un número considerable de perros que viven en la calle o que han sido abandonados en refugios, lo que se ha establecido como un problema social; también para los perros, pues muchos necesitan un lugar donde vivir, ya que subsisten en circunstancias muy precarias. Las instituciones públicas colombianas deben fomentar la opción de adoptar un perro en lugar de comprarlo. Este acto generará beneficios para los ciudadanos en general, ya que habrá menos perros en la calle. Por último, pensar en las necesidades del animal antes que en las del dueño, beneficia la relación entre ambos porque al cubrirlas, las mascotas tendrán menos problemas comportamentales.

Durante el análisis de los resultados se mencionó que este tipo de problemas parece más notable en las áreas pobres de las ciudades colombianas. Algunas de las razones de este hecho pueden deberse a que los individuos que residen en estratos bajos se enfrentan a mayores problemas, tienen menos recursos para encargarse de los perros y deben atender otras circunstancias que afectan sus vidas personales, más allá del problema canino.

No obstante, este estudio se centra en analizar si existen razones de tipo psicosocial relacionadas con el consumo y la percepción de los perros de raza en las diferentes zonas de las ciudades colombianas y, de hecho, los resultados muestran que la relación entre el materialismo y el valor social percibido de los perros de raza solo se puede aceptar entre los dueños que viven en los estratos más bajos de la ciudad.

La influencia directa del materialismo en los problemas comportamentales de los perros y en la intención de abandonarlos se puede aceptar para los ciudadanos que viven tanto en las zonas ricas de la ciudad como en las zonas más pobres. Sin embargo, a tenor de los resultados, se puede establecer que los dueños que viven en zonas más pobres tienen mayor probabilidad de abandonar al animal si perciben los problemas comportamentales que los dueños que viven en los estratos altos de la ciudad.

Estudios recientes han indicado que los ciudadanos de zonas pobres pueden mostrar tendencias incluso más materialistas que los que viven en zonas más ricas de la ciudad (Carey y Markus, 2016). La razón de una intención y motivación de adquirir productos y marcas que otorgan prestigio puede deberse a procesos de imitación (Amaral y Loken, 2016). De esta forma, las personas que viven en las zonas de bajo estrato social en Colombia pueden desear perros que muestran estatus social. Por ello, parece especialmente importante generar conciencia pública sobre las malas consecuencias de adquirir perros de raza valoración de tipo social o consumismo.

\section{Referencias}

Agudelo-Flórez P., Castro B., Rojo-Ospina, R., y Henao-Villegas, S. (2012). Canine brucellosiss: Seroprevalence and risk factors in pets from eleven neighbourhoods in Medellin, Colombia. Revista de salud pública, 14(4), 1-8.

Amaral, N. B., y Loken B. (2016). Viewing usage of counterfeit luxury goods: Social identity and social hierarchy effects on dilution and enhancement of genuine luxury brands. Journal of Consumer Psychology, 26(4), 483-495. doi: 10.1016/j. jcps.2016.02.004

Bagozzi, R. P., y Yi, Y. (1988). On the evaluation of structural equation models. Journal of the Academy of Marketing Science, 16(1), 74-94. doi: 10.1007/ BFo2723327 
Belk, R. W. (1985). Three scales to measure constructs related to materialism: reliability, validity, and relationships to measures of happiness. Advances in Consumer Research, 11, 291-297.

Bogliacino, F., Jiménez-Lozano, L. M., y Reyes-Galvis, D. A. (2015). Identificar la incidencia de la estratificación socioeconómica urbana sobre la segregación de los hogares bogotano. Investigaciones y Productos, 24, 2-163.

Buckland, E. L., Corr, S. A., Abeyesinghe, S. M., y Wathes, C. M. (2014). Prioritisation of companion dog welfare issues using expert consensus. Animal Welfare, 23, 39-46. doi: 10.7120/o9627286.23.1.039

Burroughs, J. E., y Rindfleisch, A. (2002). Materialism and Well-Being: A Conflicting Values Perspective. Journal of Consumer Research, 29(3), 348-370.

Byrne, D. (1997). An overview (and underview) of research and theory within the attraction paradigm. Journal of Social and Personal Relationships, 14(3), 417-431. doi: 10.1177/0265407597143008

Carey, R. M., y Markus, H. R. (2016). Understanding consumer psychology in working-class contexts. Journal of Consumer Psychology, 26(4), 568-582. doi: 10.1016/j.jcps.2016.08.004

Dabholkar, P. A., y Bagozzi, R. P. (2002). An attitudinal model of technology-based self-service: Moderating effects of consumer traits and situational factors. Academy of Marketing Science, 3o(3), 184197. doi: 10.1177/0092070302303001

Deckop, J. R., Jurkiewicz, L. C., y Giacalone, R. A. (2010). Effects of materialism on work-related personal well-being. Human Relations, 63(7), 1007-1030. doi: 10.1177/0018726709353953

Dogan, V. (2015). The effect of materialism and proximity of clothing to self on the ratio of feeling younger: implications for the consumption experiences of older people in Turkey. International Journal of Consumer Studies, 39, 564-573. doi: 10.1111/ ijcs.12196

Drentea, P. (2000). Age, debt and anxiety. Journal of Health and Social Behavior, 41, 437-450. doi: $10.2307 / 2676296$

Duh, H. I. (2015). Antecedents and Consequences of Materialism: An Integrated Theoretical Framework. Journal of Economics and Behavioral Studies, 7(1), 20-35. doi: 10.22610/jebs.v7i1.560.g56o

Durvasula, S., y Lysonsky, S. (2010). Money, money, money: How do attitudes toward money impact vanity and materialism? The case of young Chinese consumers. Journal of Consumer Marketing, 27(2), 169-179. doi: 10.1108/07363761011027268
Easterlin, R. A., y Crimmins, E. (1991). Private Materialism, Personal Self-Fulfillment, Family Life, and Public Interest: The Nature, Effects, and Causes of Recent Changes in the Values of American Youth. Public Opinion Quarterly, 55, 499-533. doi: $10.1086 / 269280$

Ellingsen, K., Zanella, A.J., Bjerkas, E. y Indrebo, A. (2010). The Relationship between Empathy, Perception of Pain and Attitudes toward Pets among Norwegian Dog Owners. AnthrSozoös, 23(3), 231-243.

Ergen, A., Baykan, B. G., y Turan, S. G. (2015). Effect of materialism and environmental knowledge on environmental consciousness among high school students: A study conducted in Istanbul province. International Journal of Human Sciences, 12(1), 511527. doi: 10.2752/175303710X12750451258931

Farnworth, M. J., Blaszak, K., Hiby, E. F., y Waran, N. K. (2012). Incidence of dog bites and public attitudes towards dog care and management in Samoa. Animal Welfare, 21, 477-486. Recuperado de http:// hdl.handle.net/10652/2278

Fernández, J., Bello, F., López, M. C., Moncada, L. I., Vargas, J. J., Ayala, M. S., Nicholls, R. S., y Lozano, C. A. (2006). Seroprevalencia de leishmaniosis visceral canina en la comuna 8 de Neiva y en cuatro municipios de Huila, Colombia. Biomédica, 26(Sup1), 121-130. doi: 10.7705/biomedica.v26i1.1506

Fornell, C., y Larcker, D. (1981). Evaluating structural equations models with unobservable variables and measurement error. Journal of Marketing Research, 18, 39-50. doi: 10.2307/3150979

Fraser, D., Weary, D. M., Pajor, E. A., y Milligan, B. N. (1997). A scientific conception of animal welfare that reflects ethical concerns. Animal Welfare, 6, 187-205. Recuperado de https://animalstudiesrepository.org/ethawel/1/

Gallego, J., Gutiérrez, L., López, D., y Sepúlveda, C. (2014). Alternativas de desmonte de la estratificación socioeconómica en el Distrito Capital. En Los límites de la estratificación. Bogotá: Editorial Universidad del Rosario.

Gallo, L. C., Bogart, L. M., Vranceanu, A., y Matthews, K. A. (2005). Socioeconomic status, resources, psychological experiences, and emotional responses: A test of the reserve capacity model. Journal of Personality and Social Psychology, 88, 386-399. Recuperado de https://pdfs.semanticscholar.org/2of1/ooo68e405e16d1fe8affba17b6b63669a552.pdf

Ger, G., y Belk, R. W. (1996). Cross-cultural differences in materialism. Journal of Economic Psychology, 17, 55-77. doi: 10.1016/0167-4870(95)ooo35-6 
Harnish, R. J., y Bridges, K. (2014). Compulsive Buying: The Role of Irrational Beliefs, Materialism, and Narcissism. Journal Rational-Emotional Cognitive-Behavior Theory, publish online. doi: 10.1007/ s10942-014-0197-0.

Hatcher, L. (1994). A Step by Step Approach to Using the SAS System for Factor Analysis and Structural Equation Modeling. SAS Institute Inc, Cary.

Hsu, Y., Liu, L., y Serpell, J. A. (2003). Dog Keeping in Taiwan: Its Contribution to the Problem of Free-Roaming Dogs. Journal of Applied Animal Welfare Science, 6(1), 1-23. doi: 10.1207/ S15327604JAWSo601_01

Inglehart, R. (1990). Culture shift in advanced industrial society. Princeton, NJ: Princeton University Press.

Karahanna, E., y Straub, D. W. (1999). The psychological origins of perceived usefulness and ease-of-use. Information and Management, 35(4), 237-250. doi: 10.1016/So378-7206(98)ooog6-2

Kasser, T., y Ryan, R. M. (1993). A Dark Side of the American Dream: Correlates of Financial Success as a Central Life Aspiration. Journal of Personality and Social Psychology, 65, 410-422. doi: 10.1016/ So378-7206(98)ooog6-2

Kilbourne, W. E., y LaForge M. C. (2010). Materialism and Its Relationship to Individual Values. Psychology \& Marketing, 27(8), 780-798. doi: 10.1002/mar.20357

Kim, D., y Jang, S. (2014). Symbolic Consumption in Upscale Cafés: Examining Korean Gen Y Consumers' Materialism, Conformity, Conspicuous Tendencies and Functional Qualities. Journal of Hospitality \& Tourism Research, 38, 39-47. doi: 10.1177/1096348014525633

Kim, H. C., y Kramer, T. (2015). Do Materialists Prefer the "Brand-as-Servant"? The Interactive Effect of Anthropomorphized Brand Roles and Materialism on Consumer Responses. Journal of Consumer Research, 42(2), 284-299. doi: 10.1093/jcr/ucvo15

King, T., Marston, L. C., y Bennett, P. C. (2012). Breeding dogs for beauty and behaviour: why scientists need to do more to develop valid and reliable behaviour assessments for dogs kept as companions. Applied Animal Behaviour Science, 137, 1-12. doi: 10.1016/j. applanim.2011.11.016

Kozinets, R., Patterson, A., Ashman, R. (2016). Networks of Desire: How Technology Increases Our Passion to Consume. Journal of Consumer Research, 43(5), 659-682. doi: 10.1093/jcr/ucw 061

Kuo, Y., y Yen, S.N. (2009). Towards an understanding of the behavioral intention to use $3 \mathrm{G}$ mobile value-added services. Computers in Human Behavior, 25, 103-110. doi: 10.1016/j.chb.2008.07.007
Liao, J., y Wang, W. (2009). Face as a mediator of the relationship between material value and brand consciousness. Psychology \& Marketing, 26(11), 987-1001. doi: 10.1002/mar.20309

Luna-Arocas, R. (2001). El consumo y la identidad: un proceso de autocreación. Investigación y marketing, 70, 6-15. Recuperado de http://www.aedemo. es/aedemo3/socios/revista70/ad-70-01.pdf

Luna-Cortés, G. (2018a). The influence of materialism on purebred dogs' welfare among two different generations in Colombia (South America). Journal of Applied Animal Welfare Science, 22(2), 149-158. doi: 10.1080/10888705.2018.1452019

Luna-Cortés, G. (2018b). Influence of materialism and self-congruity on the relationship of human with their companion dogs. Social Marketing Quarterly, 24(4), 249-263. doi: 10.1177/1524500418801002

Mason, G., y Mendl, M. (1993). Why is there no simple way of measuring animal welfare? Animal Welfare, 2, 301-319. Recuperado de http://hdl.handle. net/10214/4673

McGreevy, P. D., y Nicholas, F. W. (1999). Some practical solutions to welfare problems in dog Breeding. Animal Welfare, 8, 329-341. Recuperado de http:// sydney.edu.au/vetscience/research/disorders/documents/solutiondogbreeding.pdf

Medina, C., Morales, L., Bernal, R., y Torero, M. (2007). Stratification and Public Utility Services in Colombia: Subsidies to Households or Distortion of Housing Prices? Economía, 7(2), 41-99. Recuperado de http://www.jstor.org/stable/20060492

Nunnally, J. C., y Bernstein I. H. (1994). Psychometric Theory. McGraw-Hill, New York.

Oakes, J. M., y Rossi, R. H. (2003). The measurement of SES in health research: Current practice and steps toward a new approach. Social Science and Medicine, 56, 769-784. Retrieved from http:// dx.doi.org/10.1016/So277-9536(02)0oo73-4

Pardo, A., Pérez, C., Góngora, A., Gómez, L., y Moreno, A. (2009). Exploratory survey of Brucella Suis infection in dogs from Villavicencio-Colombia. Revista MVZ Córdoba, 14(2), 1-6.

Paternina-Gómez, M., Díaz-Olmos, Y., Paternina, L. E., y Bejarano, E. E. (2013). Alta prevalencia de infección por Leishmania (Kinetoplastidae: Trypanosomatidae) en perros del norte de Colombia. Biomédica, $33,375-82$.

Patronek, G. J., Glickman, L. T., y Moyer, M. R. (1995). Population dynamics and the risk of euthanasia for dogs in an animal shelter. Anthrozoös, 8, 31-43. doi: 10.2752/o89279395787156455 
Piff, P., Kraus, M. K., Côté S., Hayden, B., y Keltner, D. (2010). Having Less, Giving More: The Influence of Social Class on Prosocial Behavior. Journal of Personality and Social Psychology, 99(5), 771-784. doi: 10.1037/aoo20092

Podoshen, J. S., y Andrzejewski, S. (2012). An examination of the relationships between materialism, conspicuous consumption, impulse buying and brand loyalty. Journal of Marketing Theory and Practice, 20(3), 319-333. doi: 10.2753/MTP10696679200306

Reese, J. F. (2005). Dogs and dog control in developing countries. Washington, DC: Humane Society Press.

Richins, M. L., y Chaplin, L. N. (2015). Material Parenting: How the Use of Goods in Parenting Fosters Materialism in the Next Generation. Journal of Consumer Research, 41(6), 1333-1357. doi: 10.1086/680087

Richins, M. L. y Dawson, S. (1992). A Consumer Values Orientation for Materialism and Its Measurement: Scale Development and Validation. The Journal of Consumer Research, 19(3), 303-316.

Roets, A., Van Hiel, A., y Cornelis, I. (2006). Does materialism predict racism? Materialism as a distinctive social attitude and a predictor of prejudice. European Journal of Personality, 20, 155-168. doi: 10.1002/per.573

Rooney, N. J., y Sargan, D. R. (2010). Welfare concerns associated with pedigree dog breeding in the UK. Animal Welfare, 19(5), 133-140.

Rose, G. M., Bakir, A., y Gentina, E. (2016). Money meanings among French and American adolescents. Journal of Consumer Marketing, 33(5), 364-375. Recuperado de https://www.researchgate.net/ publication/233670946_Welfare_concerns_associated_with_pedigree_dog_breeding_in_the_UK

Ruíz, J. D., Giraldo, C. A., López, V. L., y Chica, J. F. (2010). Seroprevalencia de Brucella canis en perros callejeros del Centro de Bienestar Animal "La Perla”, Medellín (Colombia). Revista Colombiana de Ciencia Pecuaria, 23(2), 1-8. Recuperado de http:// www.redalyc.org/pdf/2950/295023450005.pdf

Salman, M. D., New, J. G., Scarlett, J. M., Kass, P. H., Ruch-Gallie, R., y Hetts, S. (1998). Human and animal factors related to the relinquishment of dogs and cats in 12 selected animal shelters in the United States. Applied Animal Behaviour Science, 1, 207-226. doi: 10.1207/s15327604jaws0103_2

Segal, B., y Podoshen, J. S. (2012). An examination of materialism, conspicuous consumption and gender differences. International Journal of Consumer Studies, 37(2), 189-198. doi: 10.1111/j.14706431.2012.01099.X
Segev, S., Shoham, A., y Gavish, Y. (2015). A closer look into the materialism construct: the antecedents and consequences of materialism and its three facets. Journal of Consumer Marketing, 32(2), 85-98. doi: 10.1007/978-3-319-10912-1_8o

Shavitt, S., Jiang, D., y Cho, H. (2016). Stratification and segmentation: Social class in consumer behavior. Journal of Consumer Psychology, 26(4), 583-593. doi: 10.1016/j.jcps.2016.08.005

Sirgy, M. J., Cole, D., Kosenko, R., Meadow, L., Rahtz, D., Cicic, M., Xi, J., Yaruvat, D., Blenkhorn, D. L., y Nagpal, N. (1995). A Life Satisfaction Measure: Additional Validation Data for the Congruity of Life Satisfaction Measure. Social Indicators Research, 34, 237-259. doi: 10.2190/AG.67.2.C

Snibbe, A. C., y Markus, H. R. (2005). You can't always get what you want: Educational attainment, agency, and choice. Journal of Personality and Social Psychology, 88, 703-720. doi: 10.1037/00223514.88.4.703

Sonntag, Q., y Overall, K. L. (2014). Key determinants of $\operatorname{dog}$ and cat welfare: behaviour, breeding and household lifestyle. Revue scientifique et technique, 33(1), 213-220. Recuperado de http://www. oie.int/doc/ged/D13669.PDF

Staggs, S. L., Long, S. M., Mason, G. E., Krishnan, S., y Riger, S. (2007). Intimate partner violence, social support, and employment in the postwelfare reform era. Journal of Interpersonal Violence, 22, 345-367. doi: 10.1177/o886260506295388

Sweeney, J. C., y Soutar, G. N. (2001). Consumer perceived value: The development of a multiple item scale. Journal of Retailing, 77, 203-220. doi: 10.1016/ Soo22-4359(01)00041-0

Taylor, S., y Todd, P. A. (1995). Understanding information technology usage: A test of competing models. Information Systems Research, 6(2), 144-176. doi: 10.1287/isre.6.2.144

Uribe-Mallarino, C. (2008). Social Stratification in Bogota: From Public Politics to the Dynamics of Social Segregation. Universitas Humanística, 65, 139172. Recuperado de http://www.scielo.org.co/pdf/ unih/n65/n65ao8.pdf

Vacalopoulos, A., y Anderson, R. K. (1993). Canine behaviour problems reported by clients in a study of veterinary hospitals. Applied Animal Behaviour Science, 37, 84-95. doi: 10.1016/01681591(93)90077-3

Van Hiel, A., Cornelis, I., y Roets, A. (2010). To Have or To Be? A Comparison of Materialism- Based Theories and Self-Determination Theory as Explanatory Frameworks of Prejudice. Journal of Personality, 78(3), 1037-1070. doi: 10.1111/j.14676494.2010.00642.X 
Veer, E., y Shankar, A. (2011). Forgive me, Father, for I did not give full justification for my sins: how religious consumers justify the acquisition of material wealth. Journal of Marketing Management, 27, 547-56o. doi: 10.1080/0267257X.2010.517707

Wells, D. L., y Hepper, P. G. (2000). Prevalence of behaviour problems reported by owners of dogs purchased from an animal rescue shelter. Applied Animal Behaviour Science, 69, 55-65. doi: 10.1016/ So168-1591(oo)oo118-o
Wright, J. C., Smith, A., Daniel, K., y Adkins, K. (2007). Dog Breed Stereotype and Exposure to Negative Behavior: Effects on Perceptions of Adoptability. Journal of Applied Animal Welfare Science, 10(3), 255-265. doi: 10.1080/10888700701353956 